\title{
Yttrium Doped Barium Cerate And Zirconate Heterostructures: Deposition And Electrochemical Characterization
}

\author{
E. Di Bartolomeo ${ }^{\mathrm{a}}$, A. D’Epifanio ${ }^{\mathrm{a}}$, N. Yanga,b, A. Tebano ${ }^{\mathrm{b}}$, G. Balestrino ${ }^{\mathrm{b}}$, \\ and S. Licoccia ${ }^{a}$ \\ ${ }^{\mathrm{a}}$ Department of Chemical Science and Technology and NAST Center, University of \\ Rome Tor Vergata, Via della Ricerca scientifica 1, 00133 Rome, Italy \\ ${ }^{\mathrm{b}}$ INFM-CNR SPIN and Department of Civil Engineering and Computer Science \\ Engineering, University of Rome Tor Vergata, Via del Politecnico, 00133, Rome, Italy
}

\begin{abstract}
Epitaxial heterostructures consisting of an yttrium doped barium cerate (BCY) layer sandwiched between two yttrium doped barium zirconate (BZY) thin layers have been deposited on insulating (001) $\mathrm{MgO}$ substrates by pulsed laser deposition. The first BZY layer was aimed at improving the lattice match with the $\mathrm{MgO}$ substrate, the second at protecting the $\mathrm{BCY}$ layer. Ionic conductivity has been studied in the $300-600^{\circ} \mathrm{C}$ temperature range as a function of the BCY thickness. Due to the absence of blocking grain boundaries, heterostructures showed a conductivity larger than that of BCY pellets sintered under optimized conditions..
\end{abstract}

\section{Introduction}

Thin films have attracted considerable interest as electrolyte materials for micro solid oxide fuel cells ( $\mu$-SOFCs) operating at intermediate temperatures $\left(600^{-}-800^{\circ} \mathrm{C}\right)(1,2)$. The decrease of the operating temperature has been pursued via the development of high temperature proton conducting electrolytes $\left(\mathrm{HTPC}_{\mathrm{S}}\right)$ (3-5). Several perovskite oxides, such as doped $\mathrm{BaCeO}_{3}, \mathrm{SrCeO}_{3}, \mathrm{BaZrO}_{3}$ and $\mathrm{SrZrO}_{3}$ show proton conductivity in hydrogen and water vapour containing atmosphere (6-8). Among these materials, BCY has the largest proton conductivity in the intermediate temperature range (about $10^{-2} \mathrm{Scm}^{-}$ ${ }^{1}$ at $600^{\circ} \mathrm{C}$ ) (9). However, these compounds suffer from poor chemical stability due to degradation in the presence of acid gases $\left(e . g ., \mathrm{CO}_{2}\right)$ and steam $(10,11)$. On the other hand, the yttrium doped $\mathrm{BaZrO}_{3}(\mathrm{BZY})$ family exhibits a good chemical stability, but their proton conductivity is relatively low (about $10^{-3} \mathrm{Scm}^{-1}$ at $\left.600^{\circ} \mathrm{C}\right)(12-15)$. Therefore, a variety of attempts have been addressed to find the best compromise between high proton conductivity and high chemical stability, such as, for instance, modifying processing parameters or coupling cerates and zirconates by solid solutions of the two compounds $(12,13,16)$.These studies suggested that the average grain dimensions decrease with an increase of $\mathrm{Zr}$ content in the barium cerate oxides. As a consequence of the higher grain boundary density, a lower conductivity, relative to the pure barium cerate compound, has been observed.

Pulsed Laser Deposition (PLD) is a flexible technique suitable for depositing thin oxide films and complex heterostructures. It is gaining increasing attention for the 
fabrication of SOFC materials, due to its capability of depositing high quality films of complex oxides $(17,18)$. Recently, a number of studies have been carried out on textured BZY thin films grown by PLD $(3,5,19)$. The excellent crystalline quality of BZY films identified them as being characterized by the largest conductivity measured for such material, because of the absence of blocking grain boundaries (4).

$\mathrm{BCY}$ is a distorted perovskite (i.e. orthorhombic structure with $a=0.622 \mathrm{~nm}, b=0.624$ $\mathrm{nm}$ and $c=8.767 \mathrm{~nm}$, pseudo-cubic lattice parameter $a=0.44 \mathrm{~nm}$ ) (20). Generally, in order to obtain epitaxial films, a good crystallographic match between film and substrate is required, both for the crystallographic structures and for the lattice parameters of the unit cells. BCY films have been grown on monocrystalline (001) $\mathrm{MgO}$ and porous $\mathrm{Al}_{2} \mathrm{O}_{3}(21)$. Such a substrate choice was mostly dictated by the need of having a negligible substrate contribution to the overall conductivity (both ionic and electronic). However, while in the case of $\mathrm{Al}_{2} \mathrm{O}_{3}$ there is no match at all between film and substrate, the lattice match for $\mathrm{MgO}$ is also very poor. Namely, $\mathrm{MgO}$ has a rock salt cubic structure with a lattice parameter of $0.421 \mathrm{~nm}$ to be compared with the pseudo-perovskite structure of BCY with a lattice parameter of $0.44 \mathrm{~nm}$. As a consequence of the large crystallographic structural differences between $\mathrm{BCY}$ and $\mathrm{MgO}$, the conductivity values of the $\mathrm{BCY}$ films, directly deposited onto bare $\mathrm{MgO}$, resulted to be two orders of magnitude lower with respect to the bulk values observed in previous studies $(21,22)$.

Aiming at improving both the structural quality (and thus the proton conductivity) and the chemical stability of BCY films, we deposited, by PLD, sandwich heterostructures consisting of a $\mathrm{BaCe}_{0.8} \mathrm{Y}_{0.2} \mathrm{O}_{3-\mathrm{x}}$ layer, about 160, 480 and $800 \mathrm{~nm}$ thick, comprised between two $\mathrm{BaZr}_{0.8} \mathrm{Y}_{0.2} \mathrm{O}_{3-\mathrm{x}}$ thin layers about $30 \mathrm{~nm}$ thick. BZY has the same crystallographic structure as BCY but a smaller pseudocubic lattice parameter $(a=0.421$ $\mathrm{nm}$ ) that matches the $\mathrm{MgO}$ lattice parameter very well. The first BZY layer was aimed at releasing the structural lattice mismatch between $\mathrm{MgO}$ and $\mathrm{BCY}$, the top most BZY layer at protecting the $\mathrm{BCY}$ film from the atmosphere. The overall structural and electrochemical performance of $\mathrm{MgO} / \mathrm{BZY} / \mathrm{BCY} / \mathrm{BZY}$ heterostructures were investigated in this paper.

\section{Experimental}

$\mathrm{BCY}$ and BZY targets for the PLD growth were prepared starting from $\mathrm{Ba}\left(\mathrm{NO}_{3}\right)_{2}(3 \%$ $\mathrm{H}_{2} \mathrm{O}$, Aldrich, 99.999\%), $\mathrm{Ce}\left(\mathrm{NO}_{3}\right)_{3} \cdot 6 \mathrm{H}_{2} \mathrm{O}$ (Aldrich, 99.9\%), $\left(\mathrm{CH}_{3} \mathrm{CO}_{2}\right)_{x} \mathrm{Zr}(\mathrm{OH})_{y}$ (Aldrich) and $\mathrm{Y}\left(\mathrm{NO}_{3}\right)_{3} \cdot 6 \mathrm{H}_{2} \mathrm{O}$ (Aldrich, 99.9\%). BCY and $\mathrm{BZY}$ dense targets were finally sintered in air at $1500^{\circ} \mathrm{C}$ for $5 \mathrm{~h}$ and $1600^{\circ} \mathrm{C}$ for $8 \mathrm{~h}$, respectively.

The thin films of BCY with a $30 \mathrm{~nm}$ buffer layer and $30 \mathrm{~nm}$ cap layer of BZY were deposited by PLD on single crystalline $\mathrm{MgO}$ (001) substrates. The KrF excimer pulsed laser source $(\lambda=248 \mathrm{~nm})$ was operated at $10 \mathrm{~Hz}$, with an energy density of $3 \mathrm{~J} / \mathrm{cm}^{2}$, the substrate temperature was about $700{ }^{\circ} \mathrm{C}$ and the $\mathrm{O}_{2}$ pressure used during the deposition was about $0.1 \mathrm{~Pa}$. The PLD system was equipped with a multitarget carousel and a Reflection High Energy Electron Diffraction (RHEED) system for the in situ diagnostic of the growth. The BCY and BZY targets were mounted on the carousel and the whole heterostructure was grown in a single run without exposing individual layers to contamination from the atmosphere. In our experimental setup a deposition rate of about 
$0.22 \AA$ per laser shot was obtained for both BCY and BZY. X-ray diffraction analysis (XRD) was used to determine the targets composition and the films lattice parameters. $\mathrm{Cu}$ $\mathrm{K} \alpha$ radiation was used. Rocking curves were measured in order to estimate the film's mosaic spread. The surface morphology and the cross section of the deposited films were investigated by field-emission scanning electron microscopy (FE-SEM, LEO Supra 35).

Impedance measurements were carried out in wet $5 \% \mathrm{H}_{2}$ in $\mathrm{Ar}\left(\sim 3 \%\right.$ vol. $\left.\mathrm{H}_{2} \mathrm{O}\right)$ by using a frequency response analyzer, coupled with a dielectric interface. The temperature ranged between 300 and $630{ }^{\circ} \mathrm{C}$ and the frequency range was $5 \mathrm{MHz}-0.01 \mathrm{~Hz}$, with a 500 $\mathrm{mV}$ alternate applied voltage. An in-plane electrode configuration was used for the impedance characterization. Au paste electrodes with dimensions of $2 \mathrm{~mm} \times 5 \mathrm{~mm}$ were painted onto the film surface and then fired at $600{ }^{\circ} \mathrm{C}$.

To check the chemical stability of the BCY heterostructures, electrochemical measurements were repeated every day for 14 days.

\section{Results and Discussion}

The heterostructure growth mechanism was monitored by in-situ RHEED. Fig.1 (a) shows (from bottom to top) the RHEED patterns of the sample obtained depositing on an $\mathrm{MgO}$ substrate a BZY buffer layer and then a BCY layer. For the $\mathrm{MgO}$ substrate $(500 \mu \mathrm{m}$ thick), in agreement with the rock salt structure factor, only the $(00),(02)$ and $\left(0^{\overline{2}}\right)$ diffraction streaks are visible. The BZY buffer layer RHEED pattern showed the typical feature of a perovskite structure, where all $(O l)$ streaks are allowed. The presence of diffraction streaks in the RHEED pattern of both the BZY and BCY layers demonstrates that the growth occurs cube-on-cube in a quasi $2 \mathrm{D}$ mode. Dashed vertical lines are a guide for the eye and show that the position of $(02)$ and $\left(0^{\overline{2}}\right)$ streaks for $\mathrm{MgO}$ and BZY coincide, as expected in view of the fact that the cubic lattice parameters of these compounds are very similar. In the case of the BCY pattern, it is possible to notice that the distance between $(02)$ and $\left(0^{\overline{2}}\right)$ streaks is smaller than for BZY, as expected because of the larger BCY lattice constant. Fig.1 (b) shows a schematic sketch of the epitaxial relationship among the crystallographic cells of $\mathrm{BCY}, \mathrm{BZY}$, and $\mathrm{MgO}$. The role of the BZY buffer layer is to accommodate the structural misfit (perovskite $v s$. rock salt) with $\mathrm{MgO}$ without need for any lattice misfit accommodation. The lattice misfit is exclusively accommodated by the BZY/BCY, purely perovskitic, interface. Such a two-step process led to an improved quality of the $\mathrm{BCY}$ layer, in terms of structural properties, relative to the direct $\mathrm{MgO} / \mathrm{BCY}$ interface.

The XRD pattern (Figure 2) of the as grown BZY/BCY/BZY (with a BCY layer 800 $\mathrm{nm}$ thick $\mathrm{nm}$ ) sandwich heterostructures on $\mathrm{MgO}$ substrates shows very intense (001) peaks. No peaks from different phases or differently oriented grains can be detected in the spectra. In the inset of Figure 2 is reported a typical BCY rocking-curve around the (002) reflection. The full width at half maximum is about $0.8^{\circ}$. The in-plane lattice parameter was measured from the asymmetric (103) reflection. A value of $0.44 \mathrm{~nm}$ for the in-plane pseudocubic lattice parameter was found, in agreement with the out-of-plane value. Such a value is in good agreement with the BCY bulk lattice parameter and demonstrates that the epitaxial strain in BCY is fully relaxed. 

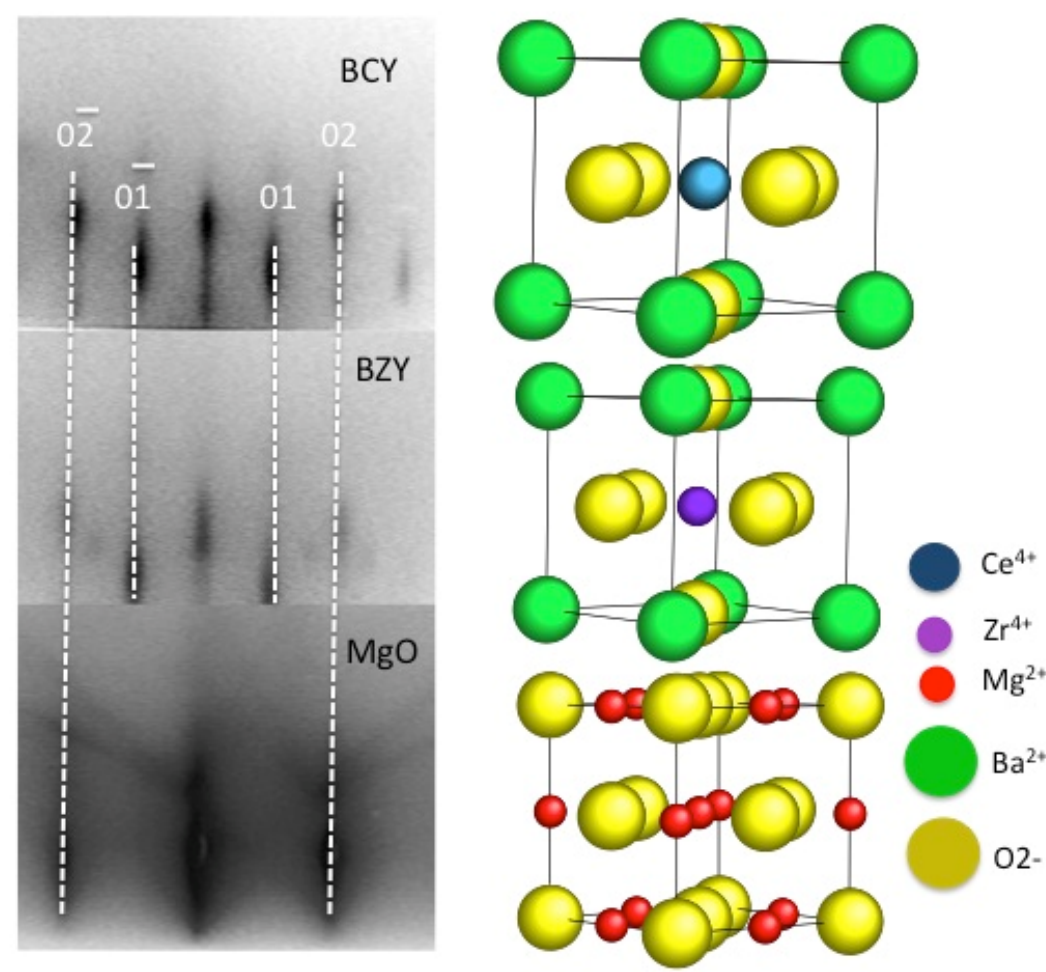

Figure 1. (a) BCY in situ RHEED pattern of $\mathrm{MgO}, \mathrm{BZY}$ and $\mathrm{BCY}$, from bottom to top, (b) schematic sketch of the structural relationship between BCY, BZY, and $\mathrm{MgO}$.

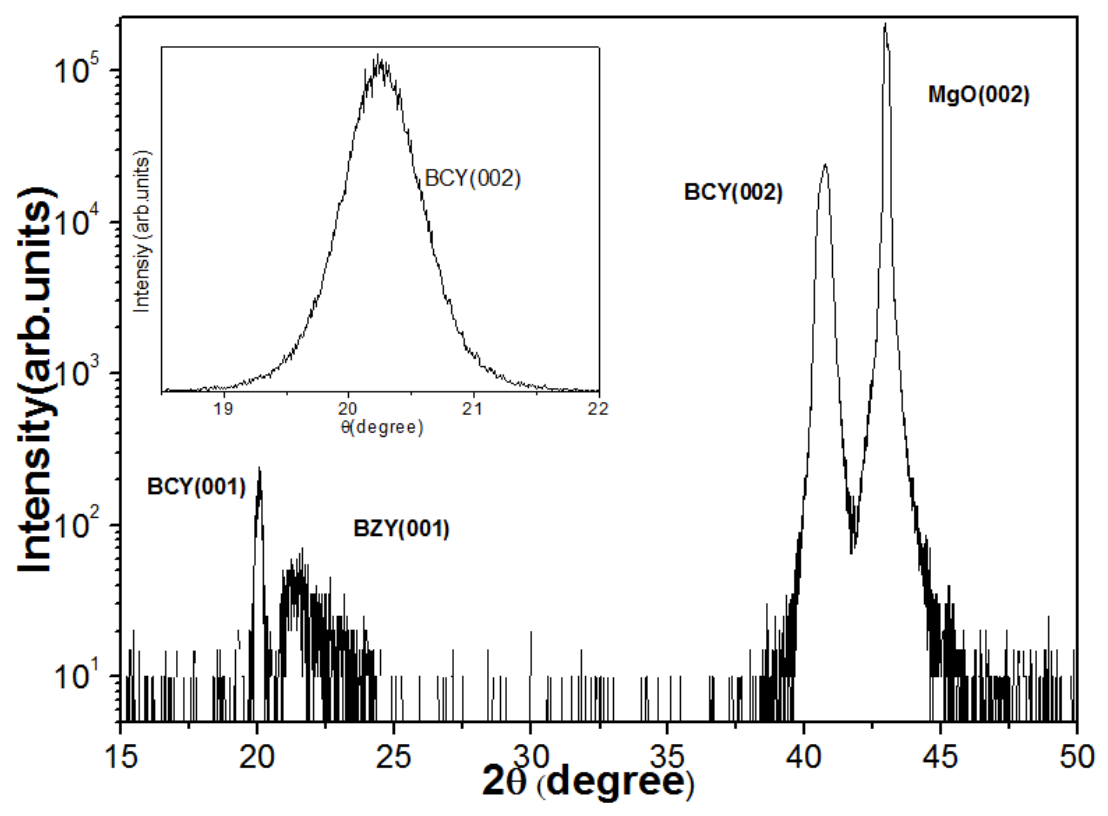

Figure 2. $\Theta-2 \Theta \mathrm{X}$ ray diffraction spectrum of a BZY/BCY/BZY heterostructure on a $\mathrm{MgO}(001)$ substrate. Beside the intense (002) peak from the substrate only $(00 l)$ peaks from BCY and BZY can be observed. The inset shows the rocking curve of the (002) $\mathrm{BCY}$ reflection. 
Figure 3 shows the FE-SEM cross section micrograph of the BZY/BCY/BZY $800 \mathrm{~nm}$ thick heterostructure where sharp interfaces between substrate and all film layers can be observed. The BCY layer fracture surface appears to be dense and uniform as typical epitaxial film growth. No grain boundary defects are detectable as it is expected for a cube-on-cube growth in a quasi 2D mode. The heterostructure surface is, except for some droplets barely visible on the top of the micrograph, quite smooth and homogeneous as typical for PLD deposition.

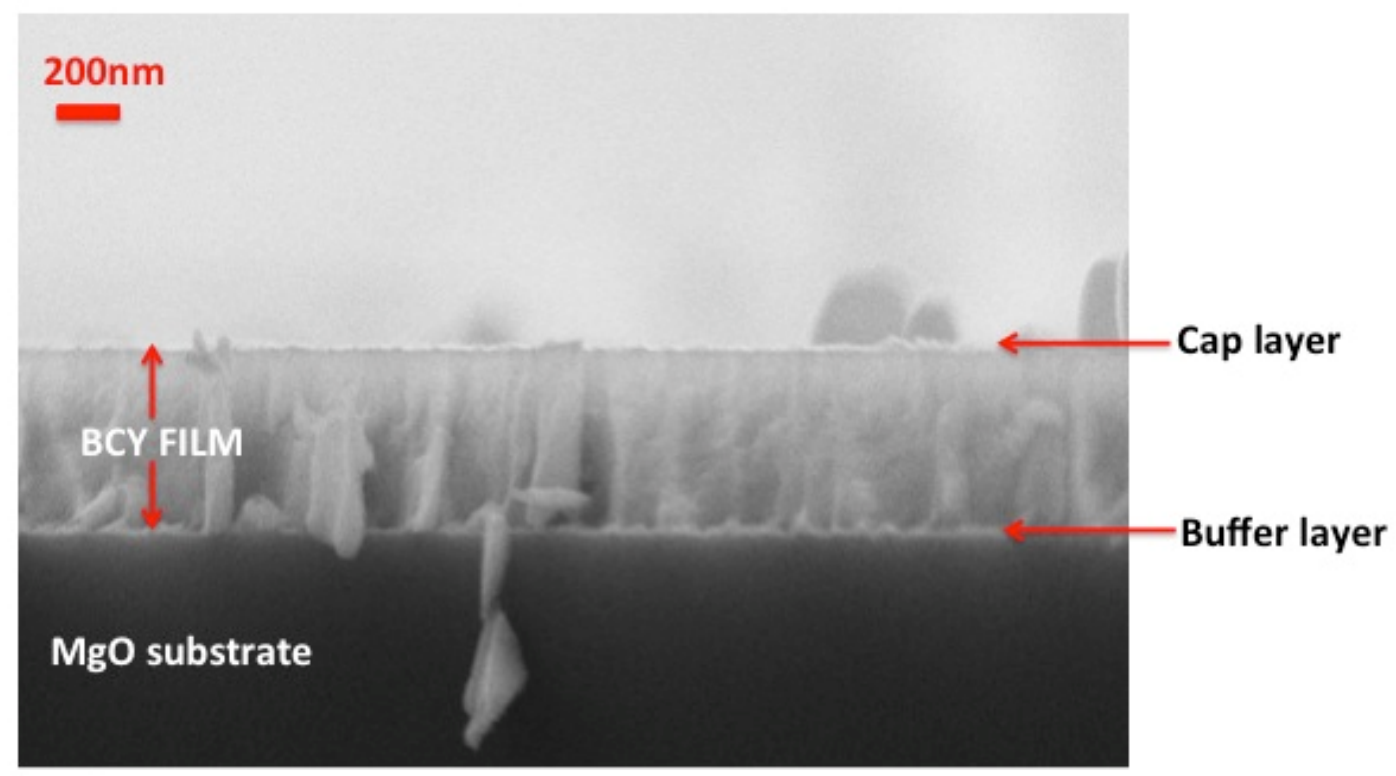

Figure 3. FE-SEM image of a BCY heterostructure's cross-section.

The ionic conductivity of the BCY sandwich heterostructures was investigated by Electrochemical Impedance Spectroscopy (EIS). Measurements were carried out in wet $5 \% \mathrm{H}_{2}$ in $\mathrm{Ar}$ atmosphere. It has to be noted that the $\mathrm{MgO}$ substrate resistance $\left(\stackrel{\Omega}{=} 10^{10} \Omega\right.$ at $600{ }^{\circ} \mathrm{C}$ ) is several orders of magnitude larger than the total resistance measured at the same temperature, which guarantees that the measured heterostructure resistance is dominated by the BCY film contribution (23). Figure 4(a) shows the complex impedance plots (Nyquist plots) at different temperatures of the $800 \mathrm{~nm}$ thick BCY heterostructure, shown as a representative example. A single semicircle with a negligible depression was observed at high frequencies at all temperatures in agreement with relevant literature on epitaxial thin films (24,2). The signal at low frequencies is attributable to electrode polarization. Fig.4(b) shows the spectroscopic plots of the impedance imaginary part (Bode plot). The bulk and the grain boundary contributions cannot be discriminated, Bode plots show in fact the presence of a single peak at high frequencies suggesting the presence of a single conduction mechanism.

Figure 5 shows the Arrhenius plots for BZY/BCY/BZY sandwich heterostructures of different thickness together with data relative to a BCY sintered pellet reported as reference (13). For all heterostructures, conductivity was slightly higher in the whole range of temperature investigated, reaching a maximum value of $1.1 \times 10^{-2} \mathrm{Scm}^{-1}$ at $630{ }^{\circ} \mathrm{C}$ for the heterostructure with the BCY layer $800 \mathrm{~nm}$ thick. 
At variance with what previously observed for $\mathrm{La}_{0.8} \mathrm{Sr}_{0.2} \mathrm{Ga}_{0.8} \mathrm{Mg}_{0.2} \mathrm{O}_{3}$ (LSGM) thin films deposited by PLD on the same substrate, conductivity was almost independent from film thickness. LSGM thin films were characterized by a highly textured columnar structure at the nanometer scale (26). Such columnar grains, coalesced during the growth so that the surface region was less defective than the interface region and correspondingly the conductivity increased with film thickness. Otherwise, the conductivity of $\mathrm{BZY/BCY/BZY} \mathrm{sandwich} \mathrm{heterostructures} \mathrm{is} \mathrm{not} \mathrm{affected} \mathrm{by} \mathrm{the} \mathrm{blocking} \mathrm{effect} \mathrm{of}$ columnar grains in agreement with the SEM observation. The activation energy value for the BCY heterostructures resulted to be very close to the value relative to sintered BCY pellets $(0.58 \pm 0.1 \mathrm{eV}$ versus $0.49 \pm 0.1 \mathrm{eV})$. The slightly improved electrochemical performance of the films can be attributed to the improved crystallographic quality.

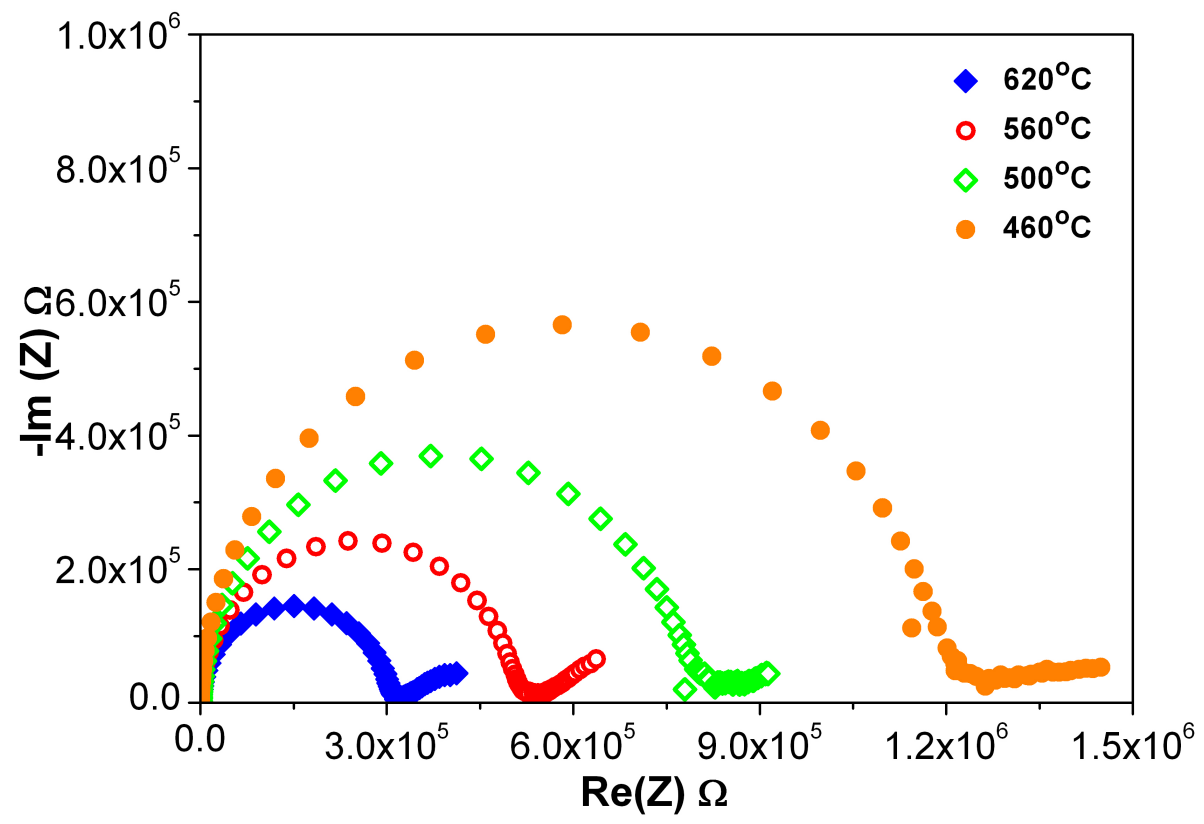

(b)

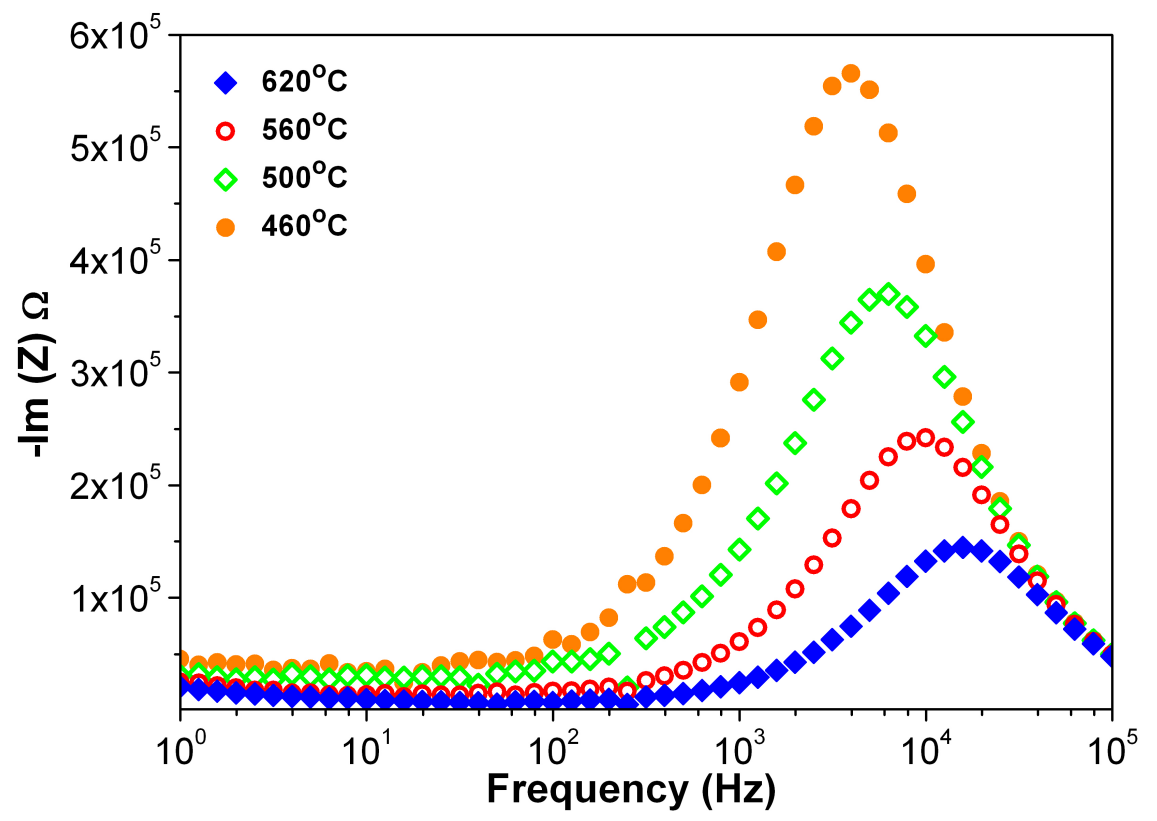

Figure 4. Temperature dependent Nyquist plots (a) and $-\operatorname{Im}(Z)$ versus frequency plots (Bode plot) (b), measured in wet $5 \% \mathrm{H}_{2}$ in Ar for a BZY/BCY/BZY heterostructure with a BCY layer $800 \mathrm{~nm}$ thick. 


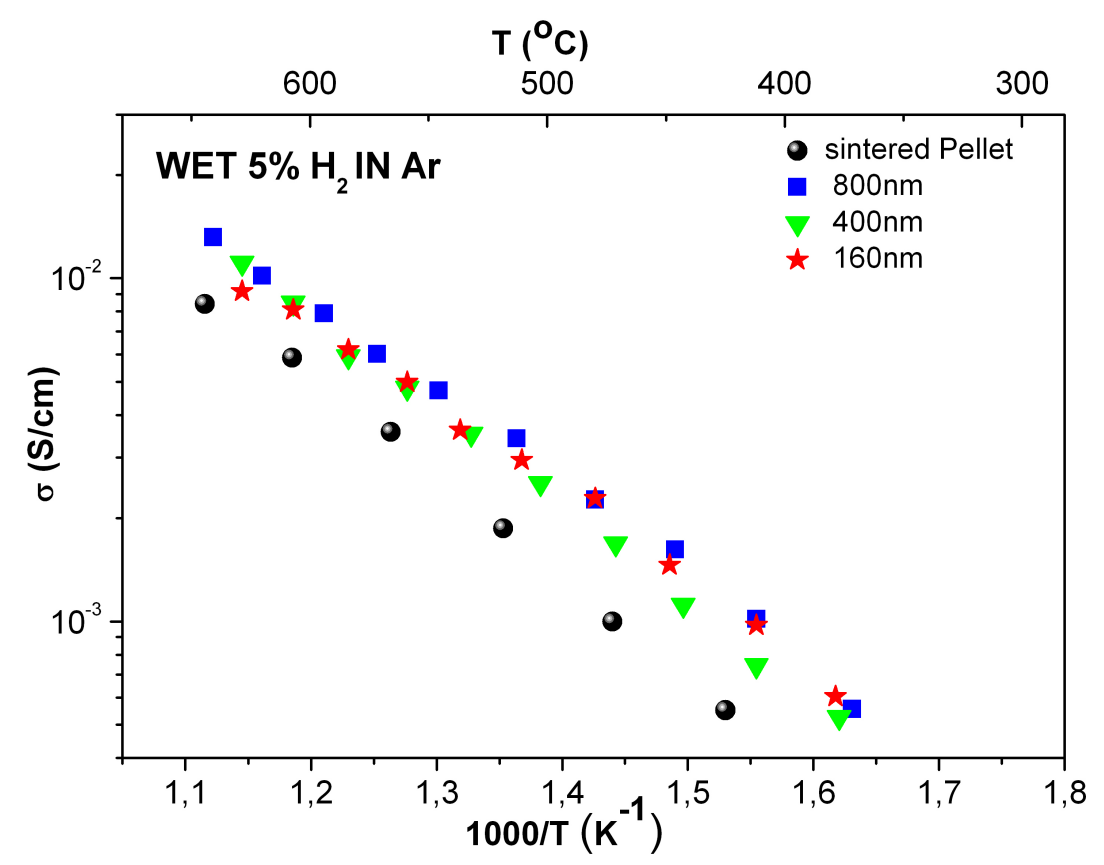

Figure 5. Electrical conductivity in wet $5 \% \mathrm{H}_{2}$ in Ar atmosphere, as a function of the temperature for three BZY/BCY/BZY heterostructures with the BCY layer $160 \mathrm{~nm}, 400$ $\mathrm{nm}$ and $800 \mathrm{~nm}$ thick, red stars, green triangles and blue squares respectively. The conductivity for the BCY sintered pellet (black circles) is also reported.

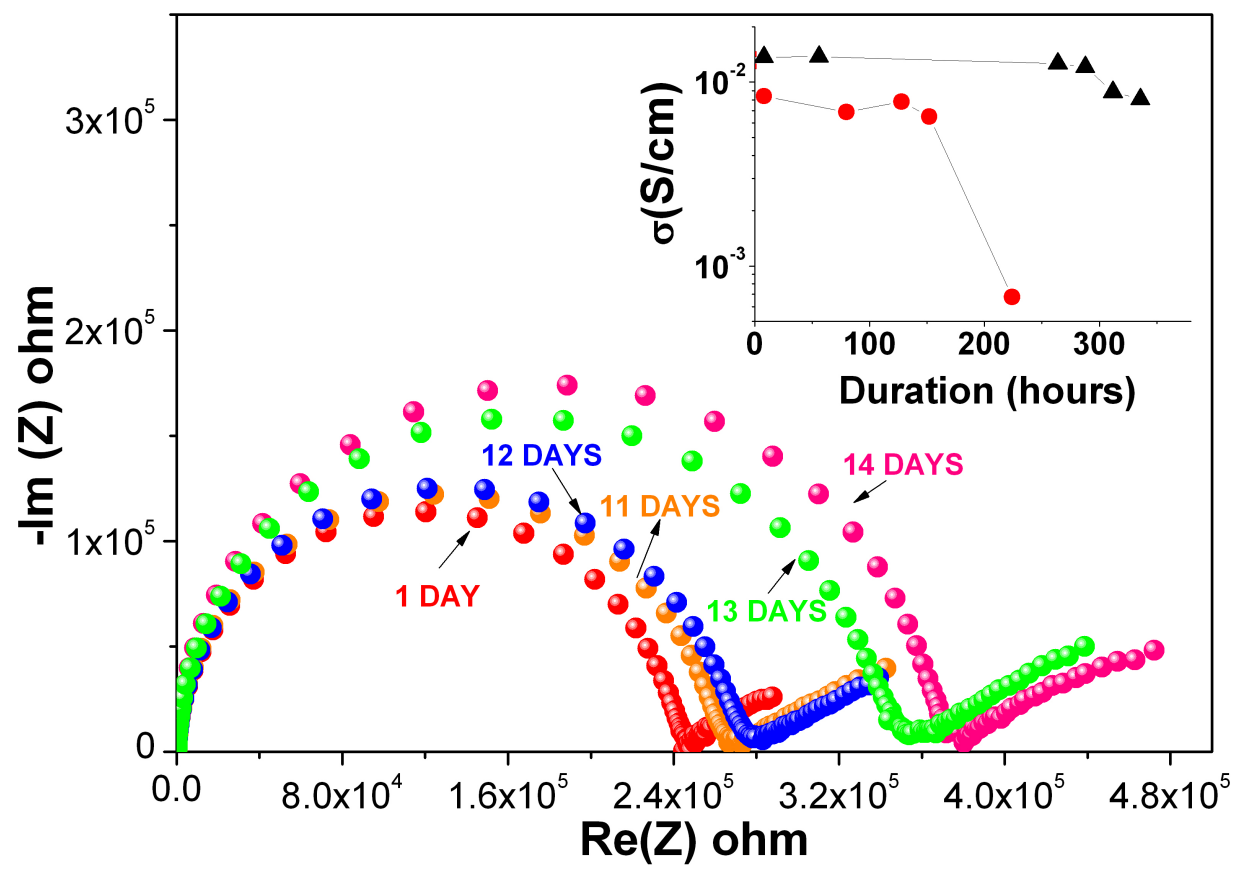

Figure 6. Nyquist plots measured over different days during the thermal stability test in operating conditions. The number next to each plot indicates the day of the test. The inset of the Fig. 6 shows the EIS thermal stability test as a function of time in hours. The black triangles represent the BCY sandwich heterostructure and the red circles represent the BCY sintered pellet. 
The chemical stability of the BZY/BCY/BZY sandwich heterostructure (with a BCY $800 \mathrm{~nm}$ thick) in wet $5 \% \mathrm{H}_{2}$ containing $\mathrm{Ar}$ atmosphere at $630{ }^{\circ} \mathrm{C}$ was investigated and compared to that of a BCY sintered pellet. Conductivity measurements were performed as a function of time. Figure 6 shows the Nyquist plots of the BZY/BCY/BZY sandwich heterostructure at $630{ }^{\circ} \mathrm{C}$ obtained at different days. The number next each plot indicates the day of testing. In the inset of Fig. 6 the conductivities of the heterostructure and of the pellet $v s$ time are reported. The heterostructure conductivity remained almost unchanged for 12 days, only a slight decrease was revealed between the $12^{\text {th }}$ day $\left(\mathrm{R}=1.2 \times 10^{-2} \mathrm{Scm}^{-1}\right)$ and the $13^{\text {th }}$ day $\left(\mathrm{R}=8.8 \times 10^{-3} \mathrm{~S}^{\mathrm{cm}-1}\right)$. For the BCY sintered pellet, a drastic decrease of the conductivity was observed on the $9^{\text {th }}$ day $\left(\sigma=8.4 \times 10^{-3} \mathrm{Scm}^{-1}\right.$ on the $1^{\text {st }}$ day and $\sigma=7 \times 10^{-}$ ${ }^{4} \mathrm{Scm}^{-1}$ on the $9^{\text {th }}$ day). From the tenth day on it was not possible to measure conductivity because of the complete degradation of the pellet. Such a result showed that the capped BCY heterostructure has an improved chemical stability in a water-containing atmosphere in comparison to the BCY sintered pellet.

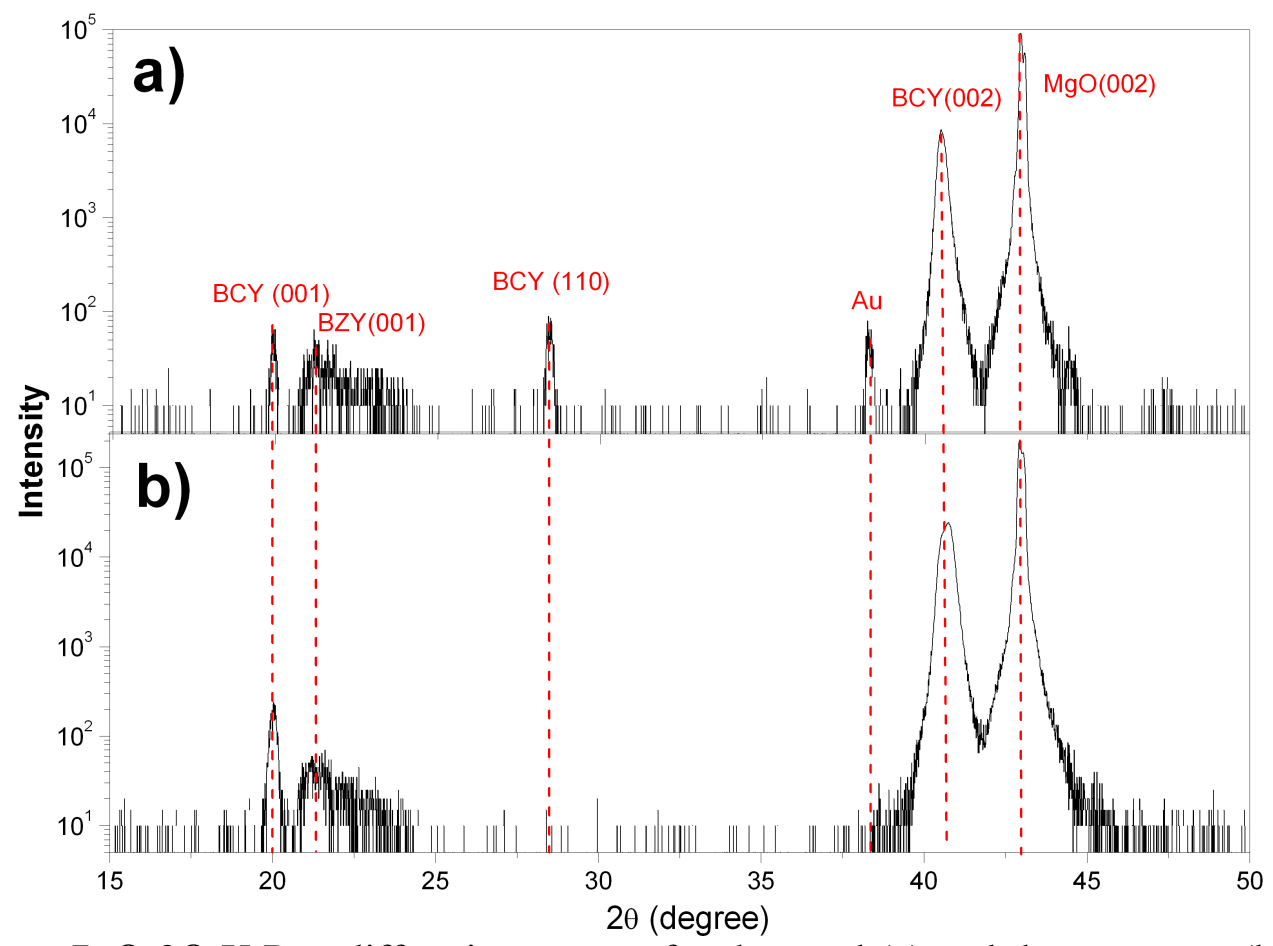

Figure 7. $\Theta-2 \Theta$ X Ray diffraction spectra for the aged (a) and the as-grown (b) BCY sandwich heterostructure.

To investigate the decrease of the conductivity observed from $13^{\text {th }}$ day of measurement in the BCY sandwich heterostructures, XRD analysis of the: (a) as-grown and (b) aged (after electrical measurements) BCY sandwich heterostructure was carried out and shown in Fig. 7. The as-grown BCY sandwich heterostructure shows (001) peaks, exclusively while the heterostructure aged for 14 days in wet $5 \% \mathrm{H}_{2}$ in $\mathrm{Ar}$ at $630{ }^{\circ} \mathrm{C}$, does not show any extra peaks such as attributable to the presence of either $\mathrm{CeO}_{2}$ or $\mathrm{Ba}(\mathrm{OH})_{2}$ phases (30). However, in the XRD pattern shown in Fig. 7 (b), it is possible to notice an intense (110) BCY peak, indicating a much stronger degree of polycrystallinity of the aged heterostructures in comparison to the as grown film. It can thus be concluded that the aging phenomenon of BCY sandwich heterostructure is not driven by chemical reactivity but by a microstructural reconstruction at high temperature $\left(630{ }^{\circ} \mathrm{C}\right)$. The 
consequent increase in the degree of polycrystallinity negatively affects the transport properties because of the occurrence of the grain boundaries contribution in the policrystalline microstructure.

\section{Conclusions}

Highly textured BZY/BCY/BZY heterostructures were grown by PLD on (001) MgO substrates. SEM and XRD investigations indicate a good crystalline quality as a result of the BZY thin buffer layer. The epitaxial growth of the films resulted in conductivity larger than that reported for BCY sintered pellets as a result of the negligible effect of perpendicular blocking grain boundaries. The heterostructure chemical stability issues have been investigated in a water-containing atmosphere at working temperatures $\left(630{ }^{\circ} \mathrm{C}\right)$. An improved chemical stability of the BCY sandwich heterostructure in comparison to sintered BCY pellets was attributed to the epitaxial BZY cap layer. However, aging in wet $5 \% \mathrm{H} 2$ in Ar caused a microstructural change from epitaxial to polycrystalline. The morphology variation led to the occurrence of grain boundaries which negatively affect the overall conductance.

\section{Acknowledgments}

The authors acknowledge the Italian Ministry of Research and Education within the program "Programmi di Ricerca Scientifica di Rilevante Interesse Nazionale-PRINAnno 2010-2011-Prot. 2010KHLKFC".

\section{References}

1. S. De Souza, S.J. Visco, and L.C. De Jonghe, Solid State Ionics, 98, 57 (1997).

2. D. Beckel, A. Bieberle-Hütter, A. Harvey, A. Infortuna, U.P. Muecke, M. Prestat, J.L.M. Rupp, L.J. Gauckler, J. Power Sources, 173, 325 (2007).

3. E.Fabbri, D.Pergolesi, A.D'Epifanio, E.DiBartolomeo, G.Balestrino, S.Licoccia and E.Traversa, Energy Environ. Sci, 1, 355 (2008).

4. D. Pergolesi, E. Fabbri, A. D'Epifanio, E. Di Bartolomeo, A. Tebano, S.Sanna, S.Licoccia, G. Balestrino and E. Traversa, Nature Material, 9, 846 (2010).

5. Y. B. Kim, T. M. Gür, H.J. Jung, S.K. Kang, R. Sinclair and F. B. Prinz, Solid State Ionics, 198, 39 (2011).

6. K.D. Kreuer and K. D. Annu. Rev. Mater. Res. 33, 333 (2003).

7. N. Bonanos, Solid State Ionics, 53-56, 967 (1992).

8. H. Iwahara, Y. Asakura, K. Katahira and M. Tanaka, Solid State Ionics, 168, 299 (2004).

9. G. Ma, T. Shimura and H. Iwahara, Solid State Ionics, 110, 103 (1998).

10. N. Zakowsky, S. Williamson and J.T.S. Irvine, Solid State Ionics, 176, 3019 (2005).

11. S.V. Bhide and A.V.J. Virkar, J. Electrochem. Soc. 146, 2038 (1999).

12. E. Fabbri, A. D'Epifanio, E. Di Bartolomeo, S.Licoccia and E. Traversa, Solid State Ionics, 179, 558 (2008). 
13. K. Katahira, Y. Kohchi, T. Shimura and H. Iwahara, Solid State Ionics, 13891 (2000).

14. P. Babilo and S.M. Haile, J. Am. Ceram. Soc. 88, 2362 (2005).

15. H. G.Bohn and T.J. Schober, J. Am. Ceram. Soc. 83, 768 (2000).

16. C. Zuo, S. Zha, M.Liu, M.Hatano and M. Uchiyama, Adv. Mater., 183318 (2006).

17. J. Yoon, R. Araujo, N. Grunbaum, L. Baqué, A. Serquis, A. Caneiro, X. Zhang, H. Wang, Appl. Surf. Sci., 254266 (2007).

18. S. Sanna, V. Esposito, D. Pergolesi, A. Orsini, A. Tebano, S. Licoccia, G. Balestrino, and E. Traversa, Adv. Funct. Mater. 19, 1713 (2009).

19. J.H. Shim, T.M.Gür and F.B.Prinz, Appl. Phys. Lett. 92, 253115 (2008).

20. D. Lin, Q. Wang, K. Peng and L.L. Shaw, J. Power Sources, 205100 (2012).

21. F.W. Dynys , M.H. Berger and A. Sayir , J. Europ. Ceram. Soc. 282433 (2008).

22. H. Hosono, T. Higuchi, and T.Hattori, J. Appl. Phys.104, 113704 (2008).

23. I. Kosacki, T. Christopher, M. Rouleaub, P.F. Bechera, J. Bentleya and D.H. Lowndesb, Solid State Ionics, 176, 1319 (2005).

24. F. Mitsugi, S. Kanazawa, T. Ohkuba, Y. Nomoto, T. Ishihara and Y. Takita, Jpn. J. Appl. Phys., 43, 299 (2004).

25. M.C. Göbel, G. Gregori and X. Guo, J. Maier, Phys. Chem. Chem. Phys., 12, 14351 (2010).

26. N. Yang, A. D’Epifanio, E. Di Bartolomeo, C. Pugnalini, A. Tebano, G. Balestrino and S. Licoccia, J. Power Sources, 222, 10 (2013) -14. 